\title{
UHPLC-ESI-HRMS Quantitation of Metabolites without Using Reference Standards: Impact of LC Flow Rate and Mobile Phase Composition on MS Responses
}

\author{
Sumithra Katragadda, Samantha Mahmoud, Dil M. Ramanathan* \\ Kean University, New Jersey Center for Science, Technology, Education \& Mathematics, Union, USA \\ Email: *ramanatd@kean.edu
}

Received August 24, 2013; revised September 25, 2013; accepted October 8, 2013

Copyright (C) 2013 Sumithra Katragadda et al. This is an open access article distributed under the Creative Commons Attribution License, which permits unrestricted use, distribution, and reproduction in any medium, provided the original work is properly cited.

\begin{abstract}
During LC-MS quantitation of drugs for pharmacokinetic assessment, usually metabolites are not quantified due to the unavailability of reference standards. If a metabolite is quantified without a reference standard, then it is assumed that the LC-MS response to a drug is similar to that of its metabolite and the standard curve, of the parent compound, is used to quantitate the metabolite. This approach could result in an over or underestimation of the metabolite. To evaluate the impact of mobile phase composition on LC-MS response, the mobile phases were interchanged between methanol, acetonitrile and a 50/50 mixture of methanol/acetonitrile. UHPLC flow rates were varied from $200-500 \mu \mathrm{L} / \mathrm{min}$, with and without the addition of reverse composition of mobile phases, at the parent drug retention time. This change was necessary to achieve uniform MS responses for drugs and their metabolites. In this study, HRMS data, obtained using orbitrap, resulted in a linear response over a wider dynamic range than that obtained using the linear ion trap. Overall, the parameters, required for achieving standard free quantitation, are dependent upon the mobile phase composition and LC flow rates.
\end{abstract}

Keywords: LC-MS; Standard Free Quantitation; HRMS; Pharmaceuticals; Metabolites

\section{Introduction}

A major component of bioanalys involves the quantitative estimation of a drug and its metabolites in biological matrices, such as plasma and urine [1,2]. When a drug is administered, mainly the enzymes, present in the liver, metabolize the drug into more polar forms to facilitate the excretion in urine or feces. The parent compound is converted into more polar forms by oxidation, reduction and hydrolysis in the phase 1 reaction and by acetylation, methylation, sulfonation and conjugation with glucuronide, sulfate, glutathione or amino acids in the phase 2 reaction [3]. The quantification of these metabolites is extremely important as sometimes the metabolites may be more pharmacologically active than the drug or at times may exert toxicity. These metabolites might be present in at extremely decreased concentrations in some cases. Hence, many analytical techniques with high sensitivity, accuracy and robustness have been developed [3-8].

Liquid chromatography coupled with mass spectrome-

${ }^{*}$ Corresponding author. try has emerged as a powerful analytical technique to detect, characterize, and quantify drug metabolites $[5,7$, 8]. To obtain quantitative information using LC-MS techniques, reference standards, of analytes, are required so that LC-MS responses from drugs and/or metabolites of unknown quantities are referenced back to LC-MS responses obtained using known quantities of drugs and/or metabolites [9]. Usually a standard curve must be generated and interpreted for each and every analyte quantified. Although a standard curve can be easily generated for the parent drug, it is often impossible to do the same for metabolites for which reference standards and internals standard are not available. Standard curve of a drug cannot be used to quantify its metabolite(s) due to the structural differences of the drug and its metabolite and a standard curve of a metabolite cannot be used to quantify another metabolite from the same drug because structural alterations, by metabolism or biotransformation, lead to unpredicted changes in ionization efficiencies, extraction efficiencies (SPE, liquid-liquid, etc) and/ or highly variable interferences from endogenous matrix 
ions [9]. However, several groups have made the simple assumption that ionization efficiencies, extraction efficiencies and LC-MS responses of a parent drug and its metabolites are similar and reported quantification of metabolites without reference standards [10-12]. On the other hand, several alternate LC-MS response corrections or response normalization methods have been explored to obtain standard free quantitation of metabolites $[13,14]$. Recently introduced regulatory guidances, related to metabolite in safety testing (MIST) [15], have further sparked interest in developing and evaluating standard free quantitation methods for quantifying metabolites [16-25].

Previously Ramanathan et al. [9] demonstrated equal responses of both drug and metabolite using response normalized liquid chromatography in combination with nanospray ionization. The authors introduced a seconddary HPLC system post column with exact reverse composition gradient to accomplish delivery of parent and its metabolite under an isocratic mobile phase condition into the mass spectrometer. These results showed an equal response of the drug and metabolite. However, this response normalization method, in combination with nanospray ionization, lacked practical aspects for routine applications in the drug metabolism laboratories. Later, the same group showed the utility of micro flow ionization techniques, captive spray ionization (CSI), for achieving equal responses for drugs and associated metabolites and reported on an option for achieving standard free quantitation $[13,26]$. Practical nature of the CSI-based approach allowed several groups to evaluate this technique for standard free quantitation [17,19,27]. Although Schadt et al. [19] and Ranasinghe et al. [27] showed that decreased flow based ESI techniques can be used for standard free quantitation, Dahal et al. [17] evaluated the inaccuracies associated with using LC-CSI-MS responses of the parent drug for quantifying metabolites. The standard curves of a parent and its metabolites, formed via O-demethylation, N-demethylation, aromatic hydroxylation and benzylic hydroxylation, were statistically different in 18 of the 20 parent-metabolite pairs regardless whether ions were formed using conventional electrospray ionization (ESI) or low-flow captive spray ionization (CSI) methods. The researchers also showed that the signal response of the drug and the metabolite is often found in four-fold differences.

Schmidt et al. [28] studied the impact of mobile phase on MS response. Using ESI-MS/MS, infrared multiphoton dissociation (IRMPD) and hydrogen-deuterium exchange (HDX), the authors also observed that when water and methanol were used as mobile phases, pAminobenzoic acid was protonated at the N-terminal end whereas when water and acetonitrile were used as mobile phases, then $70 \%$ of the protonation was observed at the
C-terminal end and $30 \%$ of the protonation was seen at the N-terminal end. Thus, in this study, it was shown that when the mobile phase composition is changed, the ionization site changes. The change in site of ionization with changes in mobile phase composition provides additional credence for the normalized response approach first reported by Ramanathan et al. [9]. Most recently, Chang et al. [29] showed that post-column infusion techniques are utilized to verify whether co-administrated drug induces any changes in matrix that affect the quantitation of a parent or its metabolites.

To further understand the impact of mobile phase and LC flow rates on MS response, experimental data were obtained using ultra high pressure liquid chromatography coupled with high resolution mass Spectrometry (UHPLCHRMS). Due to the high pressures achieved using UHPLC system, the submicron particle size columns afford faster and more efficient separation of a drug and its metabolites. When using HPLC-based methods, drastic changes in mobile compositions are required for chromatographic separation of drugs from its metabolites. On the other hand, under UHPLC conditions, the mobile phase changes, required for separation of drugs from its metabolites, are minimal and possibly minimize mobile phase induced changes in ionization sites. This, in turn, could provide an option for quantification of metabolites without reference standards.

Equimolar concentrations of dextromethorphan, tolbutamide, caffeine, primaquine and associated known metabolites, dextrorphan, 4-hydroxytolbutamide, 1,7-dimethylxanthine, 2-methylquinoxaline, respectively were used in this study (Table 1). A generic gradient method was developed to separate the drugs and its metabolite in plasma. To evaluate the parameters, such as site of ionization and mobile phase composition that could affect the standard free quantitation, different flow rates and mobile phase compositions were used. Simultaneous infusion of the reverse composition of mobile phases, introduced via ESI source auxiliary inlet, was also used to evaluate the UHPLC-MS signal responses between drugs and associated metabolites.

\section{Experimental}

\subsection{Materials}

HPLC grade water, acetonitrile, and methanol were purchased from Pharmaco-Aaper (Brookfield, CT) and acetic acid from Sigma-Aldrich (St. Louis, MO). Dextromethorphan, dextrorphan, tolbutamide, caffeine, 1,7dimethylxanthine (Paraxanthine), primiquine, and 2methylquinoxaline were also purchased from SigmaAldrich. 4-hydroxy tolbutamide was purchased from Cayman Chemicals (Ann Arbor, MI). All drugs were obtained in powder form. Stock solutions were made at 
Table 1. List of Pharmaceuticals, metabolites and corresponding UHPLC retention times, exact and mass defect information.

\begin{tabular}{|c|c|c|c|c|}
\hline Analyte Name & Structure & $\begin{array}{l}\text { Elemental } \\
\text { Composition }\end{array}$ & $\begin{array}{l}\text { Exact Mass of Monoisotopic } \\
\qquad[\mathrm{M}+\mathrm{H}]^{+}(\mathrm{Da})\end{array}$ & $\begin{array}{l}\text { UHPLC Retention } \\
\text { Time }(\min )^{\mathrm{a}}\end{array}$ \\
\hline Dextromethorphan & & $\mathrm{C}_{18} \mathrm{H}_{26} \mathrm{NO}$ & 272.2008 & 10.3 \\
\hline Dextrorphan & & $\mathrm{C}_{17} \mathrm{H}_{24} \mathrm{NO}$ & 258.1852 & 9.2 \\
\hline Caffeine & & $\mathrm{C}_{8} \mathrm{H}_{11} \mathrm{~N}_{4} \mathrm{O}_{2}$ & 195.0876 & 7.2 \\
\hline Paraxanthine & & $\mathrm{C}_{7} \mathrm{H}_{9} \mathrm{~N}_{4} \mathrm{O}_{2}$ & 181.0720 & 8.6 \\
\hline Tolbutamide & & $\mathrm{C}_{12} \mathrm{H}_{19} \mathrm{~N}_{2} \mathrm{O}_{3} \mathrm{~S}$ & 271.1111 & 9.8 \\
\hline 4-hydroxytolbutamide & & $\mathrm{C}_{12} \mathrm{H}_{19} \mathrm{~N}_{2} \mathrm{O}_{4} \mathrm{~S}$ & 287.1060 & 8.6 \\
\hline Primaquine & & $\mathrm{C}_{15} \mathrm{H}_{22} \mathrm{~N}_{3} \mathrm{O}$ & 260.1757 & 7.1 \\
\hline 2-methylquinoxaline & & $\mathrm{C}_{9} \mathrm{H}_{9} \mathrm{~N}_{2}$ & 145.0760 & 9.2 \\
\hline Fluoxetine (IS) & & $\mathrm{C}_{17} \mathrm{H}_{19} \mathrm{NOF}_{3}$ & 310.1413 & 11.2 \\
\hline
\end{tabular}

a: Mobile phase $\mathrm{A}=$ Water $(0.1 \%$ formic acid $)$ and Mobile phase $\mathrm{B}=50 / 50$ methanol $/$ acetonitrile $(0.1 \%$ formic acid $)$; UHPLC flow rate $=200 \mu \mathrm{L} / \mathrm{min}$; IS $=$ Internal standard.

equal $\mathrm{mg} / \mathrm{mL}$ concentrations with equal amounts of DMSO and acetonitrile. Standards were made at different concentrations ranging from $2500 \mathrm{ng} / \mathrm{mL}$ to $1 \mathrm{ng} / \mathrm{mL}$. A 50:50 (v/v) mixture of aqueous and organic mobile 
phases, rat, dog and human plasmas were used to prepare standards. All plasma samples were purchased from Bioreclamation, Inc (Westbury, NY).

\subsection{Sample Preparation}

To minimize matrix-related interferences, a liquid-liquid extraction procedure was used to extract the drug and associated metabolites enriched in plasma. To $100 \mu \mathrm{L}$ of drug and metabolite $50 \mu \mathrm{L}$ of internal standard (fluoxetine) was added. After 5 minutes of equilibration time, 50 $\mu \mathrm{L}$ of Ammonium bicarbonate and $500 \mu \mathrm{L}$ of Ethyl acetate were added. All tubes were shaken for 15 minutes and centrifuged for 5 minutes at $2000 \times \mathrm{g}$ rpm. The organic layer was separated and evaporated to dryness. To these tubes, $150 \mu \mathrm{L}$ of 70:30 mixtures of water and acetonitrile were added and centrifuged for 5 minutes. To check for recovery of extraction, samples, from prespiked and post-spiked aliquots, were injected into UHPLC; LC-UV and LC-ESI-MS responses were compared for semi-quantitative estimation of extraction recoveries for the analytes listed in Table $\mathbf{1 .}$

\subsection{LC-ESI-LTQ-MS (or LC-ESI-LRMS) and LC-ESI-HRMS}

All experiments were performed using a Thermo Scientific LTQ-Orbitrap Discovery mass spectrometer (San Jose, CA). The spectrometer was equipped with an electro spray ionization source operated in the positive ionization mode. The entire UHPLC eluent, from the column, was introduced into the IonMax ESI source, interfaced with an LTQ-Orbitrap. All mass spectra were acquired in the full-scan mode from $\mathrm{m} / \mathrm{z}$ (mass/charge ratio) 1001100 using the Windows-based Thermo Fisher Xcalibur software platform. For all experiments, the default data acquisition parameters (i.e. automatic gaincontrol) were used. All LTQ mass spectra were recorded with unit mass resolution (LRMS $=$ low resolution mass spectra) across the $\mathrm{m} / \mathrm{z}$ range; all orbitrap spectra were recorded with 30,000 resolution at $\mathrm{m} / \mathrm{z} 400$ (HRMS). The Spray voltage and ion transfer capillary temperature were set at $4.5 \mathrm{kV}$ and $350^{\circ} \mathrm{C}$, respectively. Sheath and auxiliary nitrogen were supplied at a flow rate of 45 and 25 arbitrary units, respectively. For each set of the drug and metabolite, a UHPLC flow rate $(200-500 \mathrm{uL} / \mathrm{min}) \mathrm{ESI}$ source and MS conditions (mainly tube lens, sheath gas, auxiliary gas) were optimized, using the corresponding drug. The source conditions were not optimized for the metabolites.

All plasma extracts and standards were analyzed using an Accela HPLC system (Thermo Fisher, San Jose, CA) consisting of a quaternary pump, a degasser, column oven and an autosampler. Chromatographic separationswere achieved using an Acquity BEH C18 (100 mm $\times$
2.1 mmi.d., $1.7 \mu \mathrm{m}$ ) (Waters Corporation, Milford, MA) analytical column. The column was maintained at room temperature. For all extracted plasma samples, the autosamplertray temperature was maintained at $4^{\circ} \mathrm{C}$. HPLC water, with $0.1 \%$ formic acid, was used as the aqueous mobile phase, and organic mobile phases were varied between 100\% acetonitrile, 100\% methanol, 50:50 (v/v) mixture of acetonitrile, and methanol with $0.1 \%$ formic acid. A $20 \mu \mathrm{L}$ aliquot of sample was injected for all analyses. The HPLC gradient, used for all the experiments, is shown in Table 2. Following LC-MS analysis, all raw data files were processed using Xcalibur; processing was based on the integrated area of the each chromatogram, which was exported to excel sheets and created a processing method with batch reprocessing sequences. All calibrations were plotted using excel.

\subsection{Reverse Composition of the Mobile Phases}

The reverse composition addition method has been described in some detail [9]. Briefly, for each set of the drug and metabolite, the mobile phase composition was calculated at the parent drug's elution retention time. Through the infusion syringe or using a separate HPLC pump, the reverse composition, of these mobile phases, was introduced into the the ESI source via the sheath of the mass spectrometer.

\section{Results and Discussion}

The first set of experiments involved evaluation of various UHPLC columns and mobile phase compositions necessary to optimally separate the 9 pharmaceuticals listed in Table 1. Calculated mass values are listed in Table 1, and all HRMS based mass measurements were routinely within $+/-5$ ppm without using any internal calibration. External calibrations were performed as needed (once a month) by infusing a mixture containing

Table 2. UHPLC gradient and mobile phase compositions used for evaluation of standard free quantitation.

\begin{tabular}{ccc}
\hline $\begin{array}{r}\text { Time } \\
(\text { min) }\end{array}$ & $\begin{array}{c}\text { Mobile Phase A } \\
\text { (Water with 0.1\% formic acid) }\end{array}$ & $\begin{array}{c}\text { Mobile Phase B Acetonitrile } \\
\text { or Methanol or 50/50 } \\
\text { Methanol/Acetonitrile } \\
\text { (with 0.1\% formic acid) }\end{array}$ \\
\hline 0.00 & 99 & 1 \\
0.10 & 99 & 1 \\
5.50 & 85 & 15 \\
11.00 & 5 & 95 \\
13.40 & 5 & 95 \\
13.50 & 99 & 1 \\
15.00 & 99 & 1 \\
\hline
\end{tabular}


caffeine, L-methionyl-arginyl-phenylalanylalanine acetate (MRFA), and Ultramark 1621 was purchased from ThermoFisher. Since analyte $\mathrm{m} / \mathrm{z}$ values were not preselected, the accuracy and precision of HRMS for quantification depended on the mass tolerance or the extracted ion chromatogram window. Tightening the mass extraction window (MEW) from 50 to $10 \mathrm{ppm}$ improved the signal-to-noise $(\mathrm{S} / \mathrm{N})$ ratio and allowed filtering of most of the endogenous/matrix ions. This ratio improved the accuracy and precision for detecting theanalyte of interest. In a recent publication Xia et al. [30] systematically evaluated thedependency of MEW on mass resolving power and discussed the merits and limitations associated with acquiring HRMS data using profile- and centroidmodes. Among the findings reported, a mass resolution of $>20,000$ and a MEW $<20 \mathrm{ppm}$ were reported to be sufficient for detecting pharmaceuticals present in plasma. In the current experiment, a mass resolving power of 30,000 at m/z 400 and a MEW of $10 \mathrm{ppm}$ filtered the majority of the endogenous signal in an effort to improve $\mathrm{S} / \mathrm{N}$ ratio. This adjustment was necessary in the selectivity and specificity for the detection and quantification of pharmaceuticals present in water. For all LTQ experiments or low resolution mass spectrometry (LRMS), unit mass resolving power was used.

Since all the pharmaceuticals and associated metabolites listed in Table 1 ionized well in the positive ionization mode, mobile phase compositions, suitable for positive ionization mode,were selected. In this study, the aqueous mobile phase was water with $0.1 \%$ formic acid for all the experiments, while the organic mobile phase (mobile phase B) was varied as shown in Table 2. Valaskovic et al. [31] showed that differences in ionization efficiencies and LC-MS responses, among various analytes, were minimized by reducing the flow rate below $10 \mathrm{~nL} / \mathrm{min}$. Other research groups have shown that equimolar response is achievable even at micro flow ranges [26]. Since both micro- and nano-flow LC separations are still unacceptable as main stream methods, anevaluation of the impact with regard to conventional LC flow rates on MS response was made via varying the UHPLC flow rate from $200 \mu \mathrm{L} / \mathrm{min}$ to $500 \mu \mathrm{L} / \mathrm{min}$. Since the mobile phase compositions and LC flow rates were varied, the elution times or retention times, of the analytes,varied between mobile phases and flow rates. In Table 1, UHPLC retention times, obtained using 200 $\mu \mathrm{L} / \mathrm{min} \mathrm{LC}$ flow rate and 50/50 methanol/acetonitrile, are reported as a point of reference.

As shown in Figures 1(a)-(c), standard curves were generated for dextromethorphan and its metabolite dextrorphan. To evaluate the method, standards, prepared with the mobile phases, were analyzed by HRMS. While maintaining the aqueous mobile phase the same (water with $0.1 \%$ formic acid), the organic mobile phase was changed from $100 \%$ acetonitrile to $100 \%$ methanol, and finally to a mixture of 50/50 methanol/ acetonitrile. In addition to changing the organic mobile phase, the LC flow rate was varied from $200 \mu \mathrm{l} / \mathrm{min}, 300 \mu \mathrm{l} / \mathrm{min}, 400$ $\mu 1 / \mathrm{min}$, and $500 \mu \mathrm{l} / \mathrm{min}$. Similar types of experiments were performed using other three sets of drugs, tolbutamide, caffeine, primiquine and corresponding metabolites respectively 4-hydroxytolbutamide, 1,7 dimethylxanthine, and 2-methylquinoxaline. Since no metabolites for primiquine were commercially available, a derivative of primiquine, 2-methylquinoxaline, was used. The standard curves for all drugs and metabolites generally showed higher signal responses at lower flow rates when compared to that of the higher flow rates. This is most likely due to the fact that higher flow rates require more sheath and auxiliary gas supply as well as higher spray voltage to minimize downstream MS contamination. Higher flow rates tend to dilute the number of ions entering the mass spectrometer ion transfer region and decrease the sensitivity. However, in the evaluation of the slope ratio, between a parent and a metabolite, a similar approach is described by Dahal et al. [17], e.g., at a flow rate of $500 \mu \mathrm{L} / \mathrm{min}$, the slope ratio is closer to 1 . As the slope ratio, between a drug and its metabolite, deviates from 1 , it is quite difficult to achieve normalized or equimolar response. Results, from this evaluation, suggest that at higher LC flow rates $(500 \mu \mathrm{L} / \mathrm{min})$, the mobile phase composition, under which a parent (dextromethorphan) and a metabolite (dextrorphan) elutes are not similar. While at lower flow rates $(200 \mu \mathrm{L} / \mathrm{min}), \mathrm{lim}-$ ited variation in the mobile phase composition resultsin limited variation in ionization leading to similar MS responses between a parent (dextromethorphan) and its metabolite (dextrorphan). Additionally, the deviation between dextromethorphan and dextrorphan slopes was greater when a mixture of 50/50 acetonitrile/methanol was used as the organic mobile phase and closer to 1 when $100 \%$ acetonitrile or $100 \%$ methanol is used as the organic mobile phase. Once again supporting the observations by Schmidt et al. [28] with respect to the impact of mobile phase on MS response, in this case, a mixture, of 50/50 acetonitrile/methanol from mobile phase B and water from mobile phase A, most likely shifts the dextromethorphan and dextrorphan ionization sites and possibly shifts the interactions between the analyte and matrix ions. Another explanation is that at higher flow rates, the presence of higher amounts of solvent molecules reduces the total available surface area and the time for an analyte to diffuse to the surface will be increased. This change in flow rate causes changes in the LC-MS response of a drug and its metabolite when present inequimolar amounts.

To extend the observations from standards in mobile phase to in vivo study samples, rat, dog and human plas- 
(a)

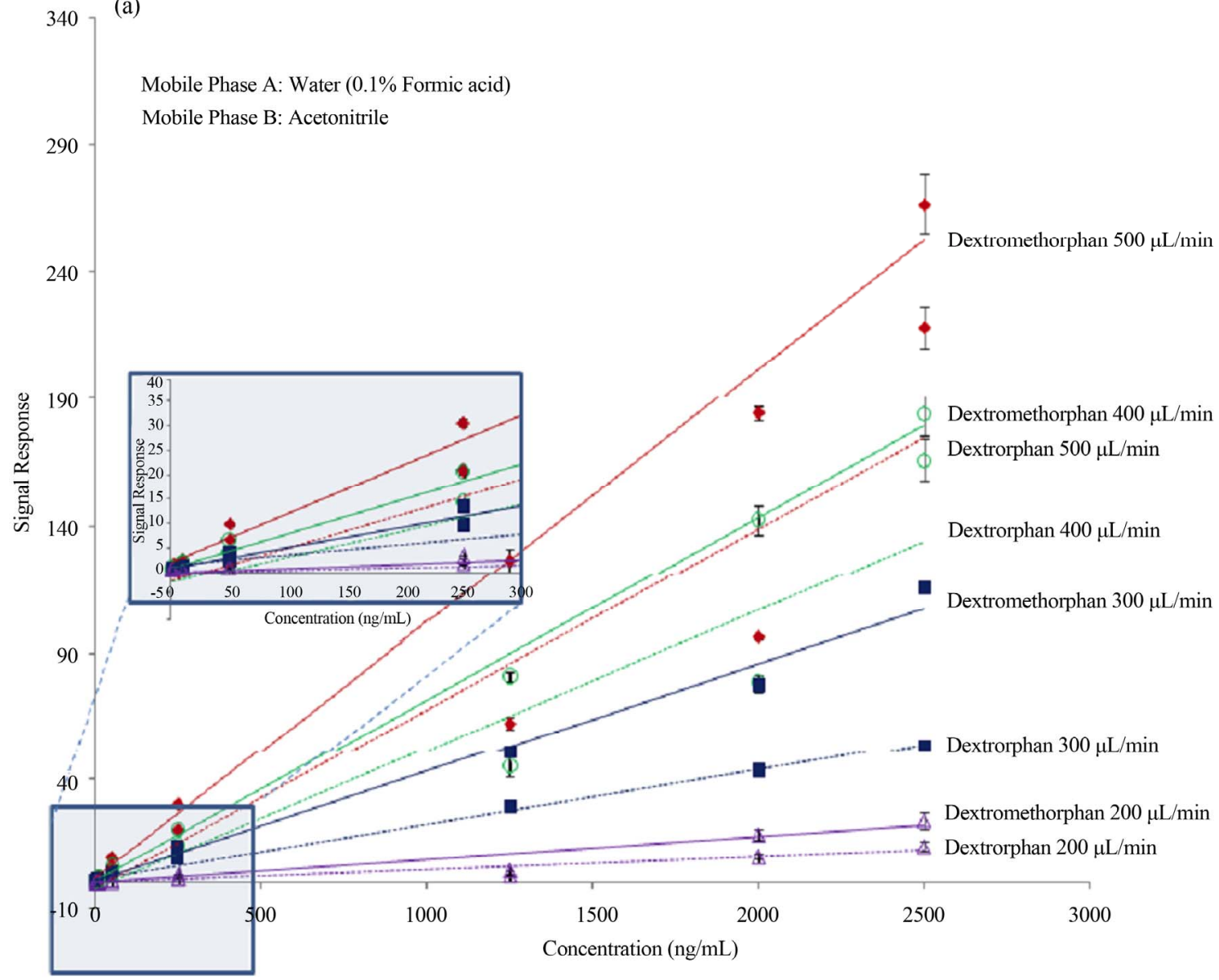

(b)

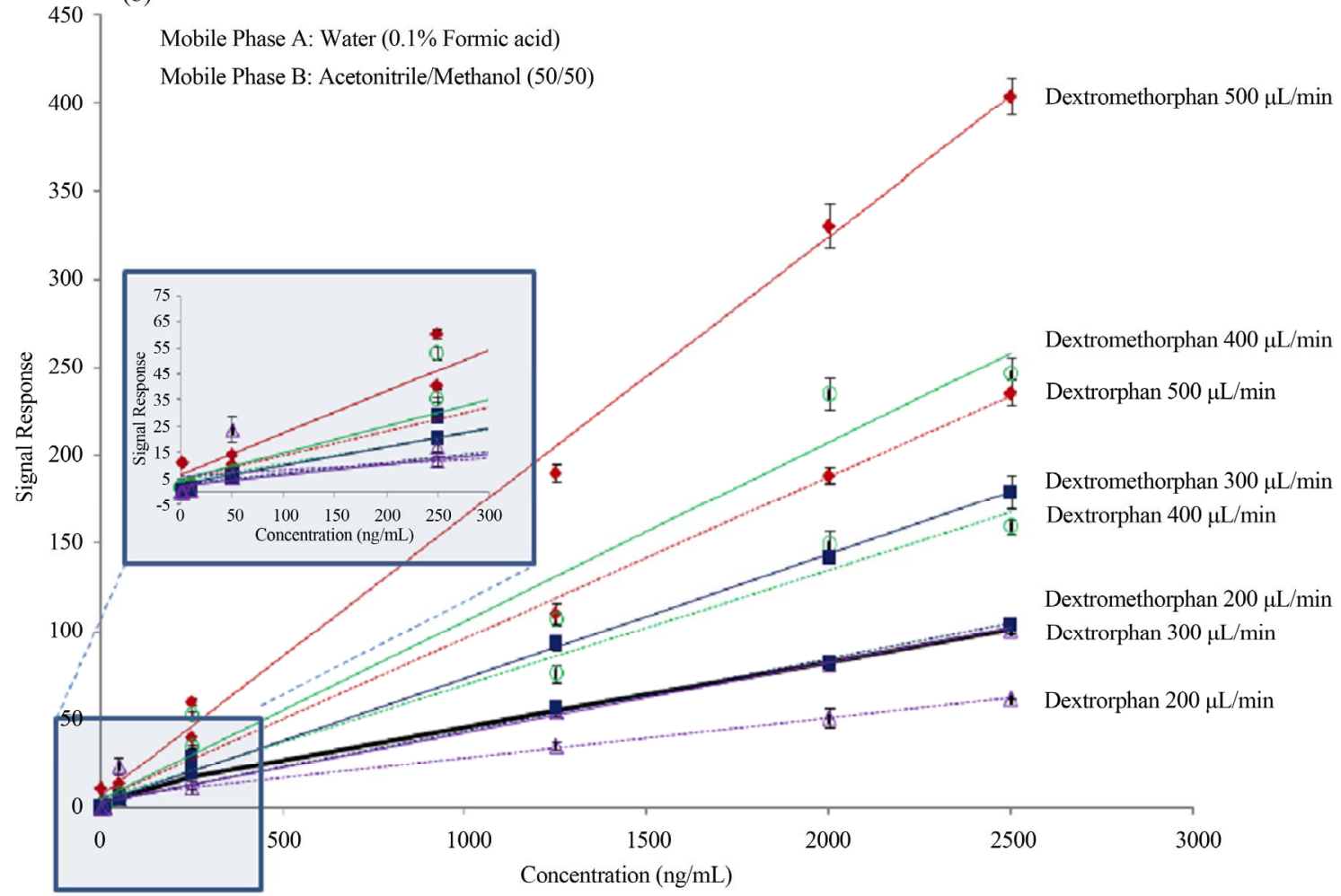




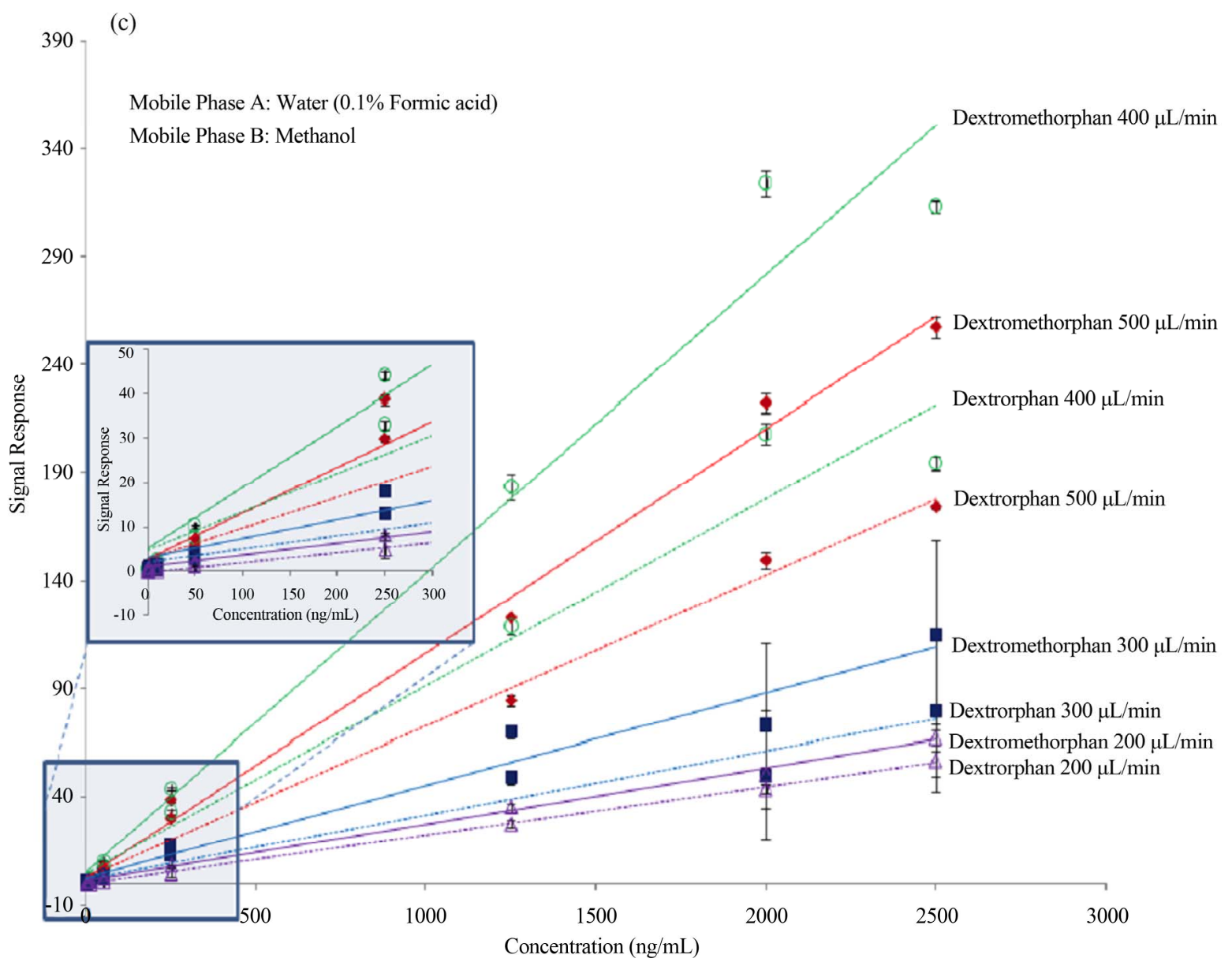

Figure 1. Standard curves of sextromethorphan and dextrorphan for all the four flow rates (a) $\mathbf{1 0 0} \%$ acetonitrile organic mobile phase; (b) 50:50 (v/v) acetonitrile and methanol organic mobile phase; (c) $100 \%$ methanol organic mobile phase.

ma were fortified with compounds listed in Table 1 and analyzed. For rat plasma samples, both linear ion trap (LRMS) and orbitrap (HRMS) were evaluated. Under LRMS full scan MS conditions, quantitation was not achievable, and non-linear standard curves were observed (Figures 2(a) and (c)). On the other hand, under HRMS conditions, standard curves were linear over a wider concentration range and the sensitivity and signal-to-noise ratio improved. Therefore, further sets, of experiments with dog and human plasma samples, were only evaluated using the orbitrap.

The organic mobile phases were varied to investigate the ionization efficiency and to possibly modify the sites of ionization. For choosing mobile phase composition, which achieves equal signal response, the difference between slopes, among the drug and the metabolite for each standard curve was calculated. In addition, all three organic mobile phase compositions in rat, dog and human plasmas were calculated as well. Ideally, the drug showed equal signal response to that of the metabolite and the difference in slope between them should result in zero. As shown in Figure 3(a), for dextromethorphan, and caffeine and associated metabolites dextrorphan, and 1,7 dimethylxanthinere spectively, the overall difference in signal response is less in $100 \%$ methanol organic mobile phase composition when compared to that of the $100 \%$ acetonitrile and 50:50 (v/v) acetonitrile and methanol. Hence, these two drugs showed a trend toward equal signal response at $100 \%$ methanol organic mobile phase composition. Additionally, marked differences, in MS response and slopes, were observed for dextromethorphan and dextrorphan in rat plasma in comparison to both being present in dog and human plasma. Examination, of the full scan HRMS data, showed absence interfering rat plasma matrix around the UHPLC elution times of dextromethorphan and dextrorphan and the larger differences in the slope was attributed to the poor extraction of dextrorphan from rat plasma.

Similarly, for primiquine and tolbutamide and associated metabolites/derivatives 2-methylquinoxaline, and 4-hydroxy tolbutamide respectively, slope differences occur lesser in $100 \%$ methanol when compared to that of the other two organic mobile phase compositions. The metabolic pathways, for these series of drugs and associ- 
(a)

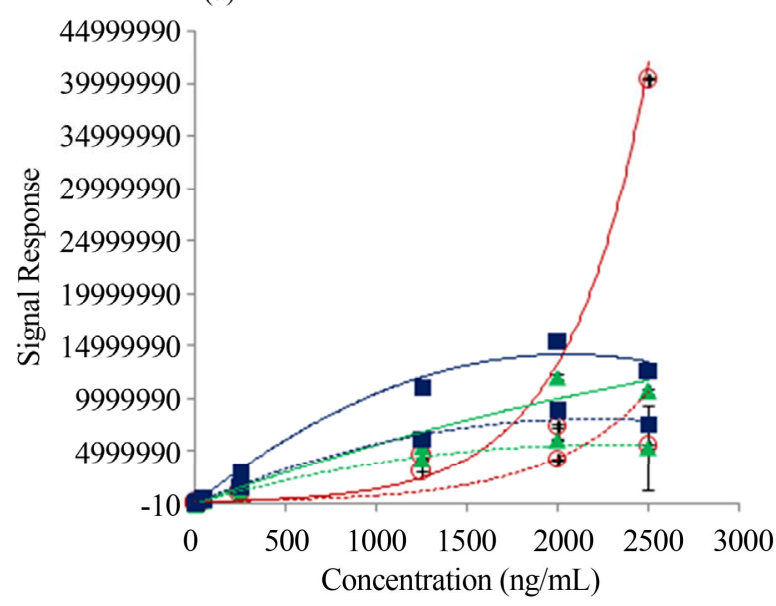

(c)

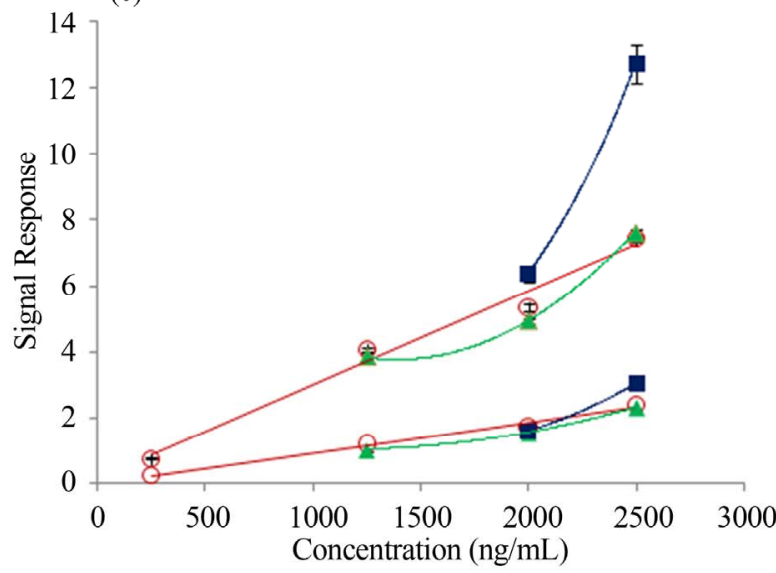

(b)

\begin{tabular}{|l}
\hline - Dextromethorphan 100\% ACN \\
- Dextromethorphan 50:50 ACN:MeOH v/v \\
- Dextromethorphan 100\% MeON \\
$\cdots$ - Dextrorphan 100\% ACN \\
-.- Dextrorphan 50:50 ACN:MeOH v/v \\
\hline
\end{tabular}

(d)

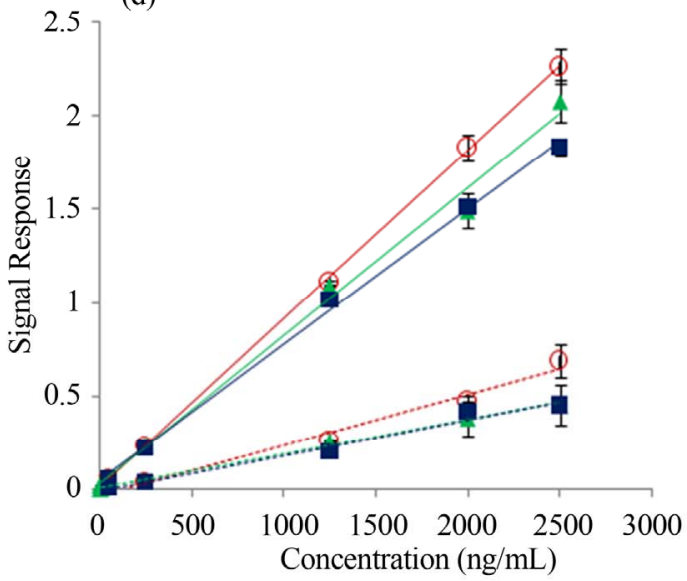

Figure 2. Comparison of linear ion trap (LRMS) and orbitrap (HRMS) standard curves for all three mobile phases at flow rate of $200 \mu \mathrm{l} / \mathrm{min}$ : (a) standard curves obtained from linear ion trap for dextromethorphan and dextrorphan; (b) Standard curves obtained from orbitrap for dextromethorphan and dextrorphan; (c) Standard curves obtained from linear ion trap for caffeine and 1,7 dimethylxanthine; (d) Standard curves obtained from orbitap for caffeine and 1,7 dimethylxanthine.

ated metabolites, included emethylation and hydroxylation. Both dextromethorphan and caffeine undergo demethylation to form dextrorphan and 1,7 dimethylxanthine pathway. As with dextromethorphan, oxygen is made available for ionization as well as interactions with solvent and matrix molecules; in caffeine, a nitrogen is exposed for similar interactions. In primiquine, the molecule is cleaved and with tolbutamide a hydroxyl group is added. Although ionization efficiencies of the drug and metabolites might be dependent on the metabolic pathway from which they were formed, all biotransformation modifications investigated, showed equal trends toward signal responses at $100 \%$ methanol organic mobile phase composition.

\section{Reverse Composition}

To achieve equal signal responses, the mobile phase 


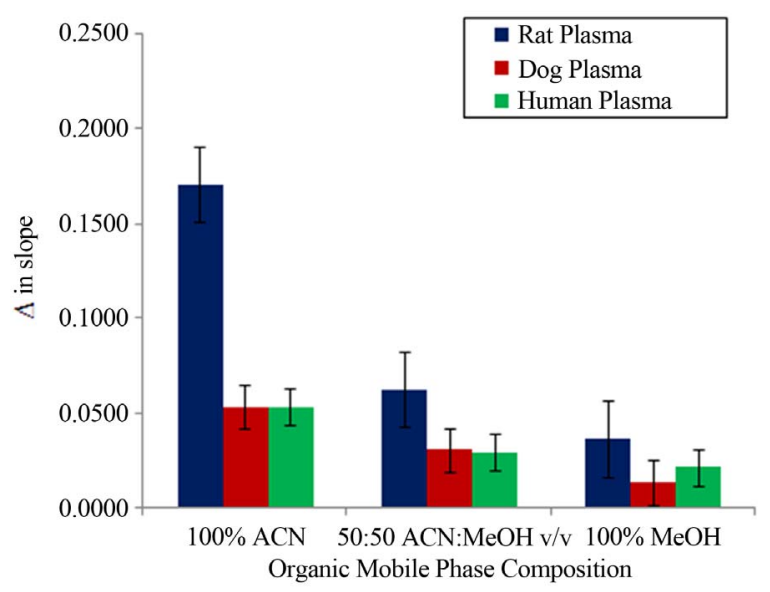

(a)

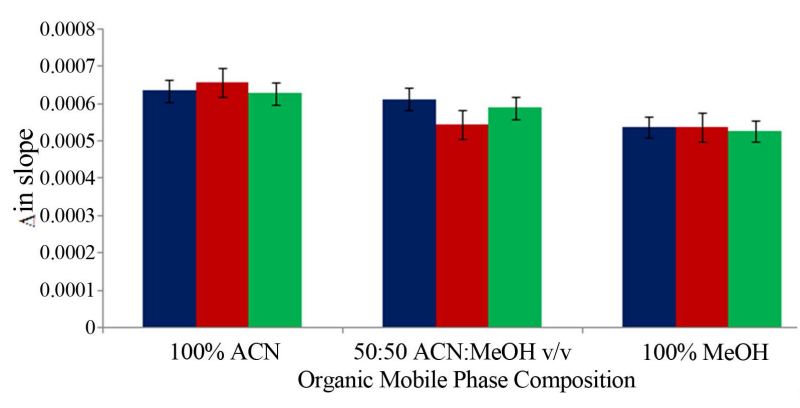

(b)

Figure 3. Comparison of all three organic mobile phases in rat, dog and human plasma extracts: (a) Difference in slope for dextromethorphan and dextrorphan; (b) Difference in slopes for caffeine and paraxanthine.

compositions, at the drug retention times, are monitored. While the mobile phase compositions are reversed, an infusion occurs through the sheath liquid inlet of the mass spectrometer. Mixing of the UHPLC mobile phase with a reverse composition provides analytes with isocratic mobile phase conditions throughout the entire UHPLC run time. The reverse gradient approach was recently described by Chang et al. [29] and previously by Ramanathan et al. [9]. Slope differences, between the drug and metabolite, were calculated for all three organic mobile phase compositions among all sets of drugs and associated metabolites. As shown in Figure 4, results from reverse gradients were compared to the slope difference obtained using conventional non-reverse gradient methods. Slope differences, for the reverse phase composition, were less when compared to that of the conventional mobile phase method. Hence, showing that infusion of reverse composition of the mobile phases can be used to obtain equal molar signal responses even at flow rates as high as $200 \mu \mathrm{L} / \mathrm{min}$.

\section{Conclusion}

The LC flow rate and mobile phase composition have an

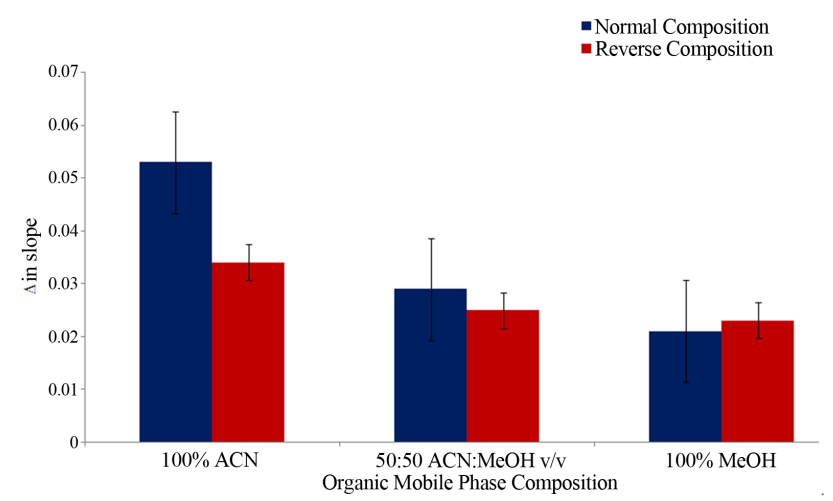

Figure 4. Infusion of reverse mobile phase composition for all three organic mobile phase compositions for evaluation for dextromethorphan and dextrorphan calibration slopes.

impact on the ionization efficiencies of the drug and associated metabolites. Hence, by altering these parameters, equal signal responses of the drug and the metabolite can be achieved. These parameters are linked to biotransformation modifications of the drug to its corresponding metabolite. This study provides additional understanding about the quantitation of metabolites in the biological matrices during early stages of drug development when reference standards of the metabolites are unavailable. Better results were obtained using HRMS due to its higher sensitivity and resolution than that of the linear ion trap. Through the reverse composition of the mobile phases at the parent drug retention time, the difference in signal responses of the drug and metabolite can further be decreased.

\section{REFERENCES}

[1] M. Jemal and Y. Q. Xia, "LC-MS Development Strategies for Quantitative Bioanalysis," Current Drug Metabolism, Vol. 7, No. 5, 2006, pp. 491-502. http://dx.doi.org/10.2174/138920006777697927

[2] W. A. Korfmacher, "Mass Spectrometry in Early Pharmacokinetic Investigations," Methods and Principles in Medicinal Chemistry, Vol. 36, 2007, pp. 401-428.

[3] M. Holčapek, L. Kolářová and M. Nobilis, "High-Performance Liquid Chromatography-Tandem mass Spectrometry in the Identification and Determination of Phase I and Phase II Drug Metabolites," Analytical and Bioanalytical Chemistry, Vol. 391, No. 1, 2008, pp. 59-78. http://dx.doi.org/10.1007/s00216-008-1962-7

[4] Y. Hsieh and W. A. Korfmacher, "Increasing Speed and Throughput When Using HPLC-MS/MS Systems for Drug Metabolism and Pharmacokinetic Screening," Current Drug Metabolism, Vol. 7, No. 5, 2006, pp. 479-489. http://dx.doi.org/10.2174/138920006777697963

[5] W. Korfmacher, "High-Resolution Mass Spectrometry Will Dramatically Change Our Drug-Discovery Bioanalysis Procedures," Bioanalysis, Vol. 3, No. 11, 2011, pp. 1169-1171. http://dx.doi.org/10.4155/bio.11.98 
[6] W. A. Korfmacher, "Principles and Applications of LC-MS in New Drug Discovery," Drug Discovery Today, Vol. 10, No. 20, 2005, pp. 1357-1367. http://dx.doi.org/10.1016/S1359-6446(05)03620-2

[7] R. Ramanathan, M. Jemal, S. Ramagiri, Y. Q. Xia, W. G. Humpreys, T. Olah and W. A. Korfmacher, "It Is Time for a Paradigm Shift in Drug Discovery Bioanalysis: From SRM to HRMS," Journal of Mass Spectrometry, Vol. 46, No. 6, 2011, pp. 595-601.

http://dx.doi.org/10.1002/jms.1921

[8] R. Ramanathan and W. Korfmacher, "The Emergence of High Resolution Mass Spectrometry as the Premier Analytical Tool in the Pharmaceutical Bioanalysis Arena," Bioanalyis, Vol. 4, 2012, pp. 501-503.

[9] R. Ramanathan, R. Zhong, N. Blumenkrantz, S. K. Chowdhury and K. B. Alton, "Response Normalized Liquid Chromatography Nanospray Ionization Mass Spectrometry," Journal of the American Society for Mass Spectrometry, Vol. 18, No. 10, 2007, pp. 1891-1899. http://dx.doi.org/10.1016/j.jasms.2007.07.022

[10] L. K. Kamdem, D. A. Flockhart and Z. Desta, "In Vitro Cytochrome P450-Mediated Metabolism of Exemestane," Drug Metabolism and Disposition, Vol. 39, No. 1, 2011, pp. 98-105. http://dx.doi.org/10.1124/dmd.110.032276

[11] D. Liu, X. Zheng, Y. Tang, J. Zi, Y. Nan, S. Wang, C. Xiao, J. Zhu and C. Chen, "Metabolism of Tanshinol Borneolester in Rat and Human Liver Microsomes," Drug Metabolism and Disposition, Vol. 38, No. 9, 2010, pp. 1464-1470.

http://dx.doi.org/10.1124/dmd.110.033381

[12] M. Shebley, U. M. Kent, D. P. Ballou and P. F. Hollenberg, "Mechanistic Analysis of the Inactivation of Cytochrome P450 2B6 by Phencyclidine: Effects on Substrate Binding, electron Transfer, and Uncoupling," Drug Metabolism and Disposition, Vol. 37, No. 4, 2009, pp. 745752. http://dx.doi.org/10.1124/dmd.108.024661

[13] R. Ramanathan, J. L. Josephs, M. Jemal, M. Arnold and W. G. Humphreys, "Novel MS Solutions Inspired by MIST," Bioanalyis, Vol. 2, No. 7, 2010, pp. 1291-1313. http://dx.doi.org/10.4155/bio.10.83

[14] Y. Yang, M. F. Grubb, C. E. Luk, W. G. Humphreys and J. L. Josephs, "Quantitative Estimation of Circulating Metabolites without Synthetic Standards by Ultra-HighPerformance Liquid Chromatography/High Resolution Accurate Mass Spectrometry in Combination with UV Correction," Rapid Communications in Mass Spectrometry, Vol. 25, No. 21, 2011, pp. 3245-3251. http://dx.doi.org/10.1002/rcm.5220

[15] USFDA, "Guidance for Industry: Safety Testing of Drug Metabolites," 2008, pp. 1-25. http://www.fda.gov/cder/guidance/6897fnl.pdf.

[16] F. Cuyckens, L. I. Balcaen, K. De Wolf, B. De Samber, C. Van Looveren, R. Hurkmans and F. Vanhaecke, "Use of the Bromine Isotope Ratio in HPLC-ICP-MS and HPLC-ESI-MS Analysis of a New Drug in Development," Analytical and Bioanalytical Chemistry, Vol. 390, No. 7, 2008, pp. 1717-1729. http://dx.doi.org/10.1007/s00216-007-1761-6

[17] U. P. Dahal, J. P. Jones, J. A. Davis and D. A. Rock,
"Small Molecule Quantification by Liquid Chromatography-Mass Spectrometry for Metabolites of Drugs and Drug Candidates," Drug Metabolism and Disposition, Vol. 39, No. 12, 2011, pp. 2355-2360. http://dx.doi.org/10.1124/dmd.111.040865

[18] R. Espina, L. Yu, J. Wang, Z. Tong, S. Vashishtha, R. Talaat, J. Scatina and A. Mutlib, "Nuclear Magnetic Resonance Spectroscopy as a Quantitative Tool To Determine the Concentrations of Biologically Produced Metabolites: Implications in Metabolites in Safety Testing," Chemical Research in Toxicology, Vol. 22, No. 2, 2009, pp. 299-310. http://dx.doi.org/10.1021/tx800251p

[19] S. Schadt, L. Z. Chen and D. Bischoff, "Evaluation of Relative LC/MS Response of Metabolites to Parent Drug in LC/Nanospray Ionization Mass Spectrometry: Potential Implications in MIST Assessment," Journal of Mass Spectrometry, Vol. 46, No. 12, 2011, pp. 1281-1286. http://dx.doi.org/10.1002/jms.2018

[20] D. A. Smith and R. S. Obach, "Metabolites: Have We MIST out the Importance of Structure and Physicochemistry?" Bioanalysis, Vol. 2, No. 7, 2010, pp. 1223-1233. http://dx.doi.org/10.4155/bio.10.92

[21] K. Vishwanathan, K. Babalola, J. Wang, R. Espina, L. Yu, A. Adedoyin, R. Talaat, A. Mutlib and J. Scatina, "Obtaining Exposures of Metabolites in Preclinical Species through Plasma Pooling and Quantitative NMR: Addressing Metabolites in Safety Testing (MIST) Guidance without Using Radiolabeled Compounds and Chemically Synthesized Metabolite Standards," Chemical Research in Toxicology, Vol. 22, No. 2, 2009, pp. 311-322. http://dx.doi.org/10.1021/tx8003328

[22] P. Wright, Z. Miao and B. Shilliday, "Metabolite Quantitation: Detector Technology and MIST Implications," Bioanalyis, Vol. 1, No. 4, 2009, pp. 831-845.

http://dx.doi.org/10.4155/bio.09.63

[23] C. Yu, C. L. Chen, F. L. Gorycki and T. G. Neiss, "A Rapid Method for Quantitatively Estimating Metabolites in Human Plasma in the Absence of Synthetic Standards Using a Combination of Liquid Chromatography/Mass Spectrometry and Radiometric Detection," Rapid Communications in Mass Spectrometry, Vol. 21, No. 4, 2007, pp. 497-502. http://dx.doi.org/10.1002/rcm.2863

[24] D. Zhang, N. Raghavan, T. Chando, J. Gambardella, Y. $\mathrm{Fu}$, D. Zhang, S. Unger and W. G. Humphreys, "LCMS/MS-Based Approach for Obtaining Exposue Estimates of Metabolites in Early Clinical Trials Using Radioactive Metabolites as Reference Standards," Drug Metabolism Letters, Vol. 1, No. 4, 2007, pp. 293-298. http://dx.doi.org/10.2174/187231207783221411

[25] M. Zhu, H. Zhang and W. G. Humphreys, "Drug Metabolite Profiling and Identification by High-Resolution Mass spectRometry," The Journal of Biological Chemistry, Vol. 286, 2011, pp. 25419-25425. http://dx.doi.org/10.1074/jbc.R110.200055

[26] R. Ramanathan, N. Raghavan, S. N. Comezoglu and W. G. Humphreys, "A Low Flow Ionization Technique to Integrate Quantitative and Qualitative Small Molecule Bioanalysis," International Journal of Mass Spectrometry, Vol. 301, No. 1-3, 2011, pp. 127-135. http://dx.doi.org/10.1016/j.ijms.2010.07.027 
[27] A. Ranasinghe, R. Ramanathan, M. Jemal, C. J. D'Arienzo, W. G. Humphreys and T. Olah, "Integrated Quantitative and Qualitative Workflow for In-Vivo Bioanalytical Support in Drug Discovery using Hybrid Q-TOF MS," Bioanalyis, Vol. 4, No. 5, 2012, pp. 511-528. http://dx.doi.org/10.4155/bio.12.13

[28] J. Schmidt, M. M. Meyer, I. Spector and S. R. Kass, "Infrared Multiphoton Dissociation Spectroscopy Study of Protonated p-Aminobenzoic Acid: Does Electrospray Ionization Afford the Amino- or Carboxy-Protonated ion?" The Journal of Physical Chemistry, Vol. 115, No. 26, 2011, pp. 7625-7632. http://dx.doi.org/10.1021/jp203829z

[29] M. Chang, Y. Li, R. Angeles, S. Khan, L. Chen, J. Kaplan and L. Yang, "Development of Methods to Monitor Ionization Modification from Dosing Vehicles and Phospholipids in Study Samples," Bioanalysis, Vol. 3, No. 15, 2011, pp. 1719-1739. http://dx.doi.org/10.4155/bio.11.164

[30] Y. Q. Xia, J. Lau, T. Olah and M. Jemal, "Targeted Quantitative Bioanalysis in Plasma Using Liquid Chromatography/High-Resolution Accurate Mass Spectrometry: An Evaluation of Global Selectivity as a Function of Mass Resolving Power and Extraction Window, with Comparison of Centroid and Profile moDes," Rapid Communications in Mass Spectrometry, Vol. 25, No. 19, 2011, pp. 2863-2878.

http://dx.doi.org/10.1002/rcm.5178

[31] G. A. Valaskovic, L. Utley, M. S. Lee and J. T. Wu, "U1tra-Low Flow Nanospray for the Normalization of Conventional Liquid Chromatography/Mass Spectrometry through Equimolarrsesponse: Standard-Free Quantitative Estimation of Metabolite Levels in Drug Discovery," Rapid Communications in Mass Spectrometry, Vol. 20, No. 7, 2006, pp. 1087-1096.

http://dx.doi.org/10.1002/rcm.2414 